\title{
The Utilization of Consignable Multi-Model in Detection and Classification of Pulmonary Nodules
}

\author{
Muhammad Bilal Zia \\ Taiyuan University of Technology \\ Taiyuan, China
}

\author{
Kamran Javed \\ Hohai Univeristy \\ Jiangsu, China
}

\author{
Zhao Juan Juan \\ Taiyuan University of Technology \\ Taiyuan, China \\ Saad Abdul Rauf \\ Taiyuan University of Technology \\ Taiyaun, China
}

\author{
Zia Ur Rehman \\ Taiyuan University of Technology \\ Taiyuan, China \\ Arooj Khan \\ Khawaja Fareed Univeristy of \\ Information and Technology \\ Pakistan
}

\begin{abstract}
Early stage Detection and Classification of pulmonary nodule diagnostics from CT images is a complicated task. The risk assessment for malignancy is usually used to assist the physician in assessing the cancer stage and creating a followup prediction strategy. Due to the difference in size, structure, and location of the nodules, the classification of nodules in the computer-assisted diagnostic system has been a great challenge. While deep learning is currently the most effective solution in terms of image detection and classification, there are many training information required, typically not readily accessible in most routine frameworks of medical imaging. Though, it is complicated for radiologists to recognize the inexplicability of deep neural networks. In this paper, a Consignable Multi-Model (CMM) is proposed for the detection and classification of a lung nodule, which first detect the lung nodule from CT images by different detection algorithms and then classify the lung nodules using MultiOutput DenseNet (MOD) technique. In order to enhance the interpretability of the proposed CMM, two inputs with multiple early outputs have been introduced in dense blocks. MOD accepts the detect patches into its two inputs which were identified from the detection phase and then classified it between benign and malignant using early outputs to gain more knowledge of a tumor. In addition, the experimental results on the LIDC-IDRI dataset demonstrate a $92.10 \%$ accuracy of CMM for the lung nodule classification, respectively. CMM made substantial progress in the diagnosis of nodules in contrast to the existing methods.
\end{abstract}

\section{Keywords}

Machine Learning, Lung Nodule Detection \& Classification, Consignable Multi-Model, LIDC-IDRI

\section{INTRODUCTION}

According to international cancer figures for 2015, about $15 \%$ of 15 million new cancer diagnoses and $18 \%$ of cancerrelevant deaths annually were lung cancer [4]. Five-year survival is about $54 \%$ for patients with an early diagnosis compared to $4 \%$ for patients with an early stage IV disease [5] if they are treated late. It is therefore quite relevant how to enhance the diagnostic accuracy for lung tumors. Early-phase treatment of lung cancer is the secret to patients ' recovery. Pulmonary nodules represent the initial indications of lung disease that can easily be recognized in low-dose CTs and have been shown to reduce pulmonary cancer mortality rates [6]. The CT diagnosis findings are also heavily dependent on clinician subjective experience and not very accurate tests. Based on the above, CAD can provide a non-intrusive approach and accurate analysis to aid radiologists to identify and classify the nodules. For the classification of the lung nodule, there are two classes of CAD: a classification technique depend on manual extraction tasks [10-12], and an automated neural extraction derived deep network [13-16]. The Lung Image Database Image Collection Dataset [7] was used in this analysis. It is led by the National Institute of Cancer (NCI), further founded by the National Institutes of Health Foundation (FNIH). In [8], a newly proposed CAD method for the detection of pulmonary nodules from pulmonary CT scans. For the test of this CAD method, LIDCIDRI was used. In To order to enhance visualizations of the relations between the artifacts of the CT scan, the system has been developed for 3D structure analysis [9] of lung Nodules. Different Detection techniques were used in [8] to identify the lung nodule. In [17], a new technique is introduced, which used multi-scale feedback and combined the features from each dense part to accomplish the classification providing 90 percent accuracy. By using a local geometric models filter, Huang et al. [18] generate Nodule candidates and increasing structural variability further by estimating the local orientation.

\section{METHODOLOGY}

In this segment, the proposed Consignable Multi-Model (CMM) has been discussed by using the Pre-processing technique to detect the lung nodule and Multi-Output DenseNet (MOD) method for the lung nodule classification. The Presented Model consists of 3 components, i.e., Input, Detection phase with different machine learning techniques, and classification part through Multi-Output DenseNet (MOD). Radiologists diagnose multi sections of a pulmonic nodule and take all the required details from the nodule into consideration. In the previous techniques, multi-view images are typical, and these images do not give full pulmonary information. Therefore, the CMM first extracts the region of interest (ROI) for the detection process, and then MOD takes the nodule location as an input to distinguish between a malignant and benign nodule for lung nodule classification. The structure of CMM is shown in Fig 1. The emphasis of the MOD technique is on small areas at the lung nodule spots annotated, and it provides two pathways through which 3D image patches are offered at different scales to cover a pulmonary nodule's locally and globally image context. Section 2.2.1 illustrates the detail of the MOD methodology. 


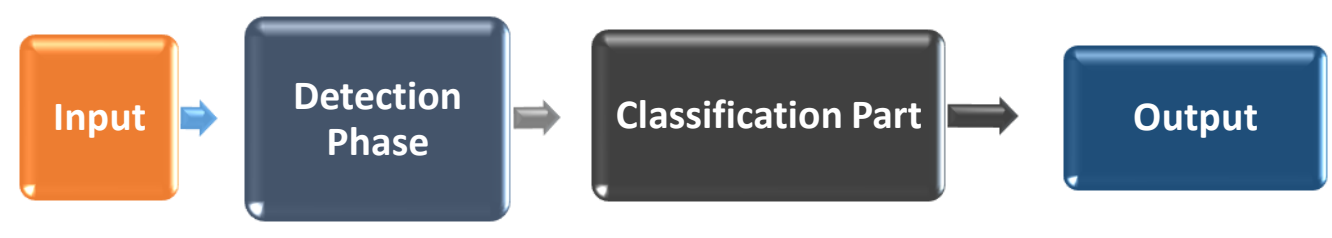

Fig 1: The Proposed Consignable Multi-Model

\subsection{Lung Nodule Detection}

The images from the Lung CT scan contains several objects, such as the lungs, vessels. So before classification, the ROI must be detected in the presented CMM. The only area in which lung nodules are present is the region of $\mathrm{k}$ concern. To order to achieve this, several forms for image processing techniques are used for lung CT scans, which is shown in Fig 2. Further methods of image processing are discussed further.

\subsubsection{Thresholding}

Thresholding is the image processing technique used for transforming gray images into binary images. Pixel $\mathrm{Ii}, \mathrm{j}$ tested for intensity with the simplest threshold methods. When $\mathrm{Ii}, \mathrm{j}$ is lesser than several fixed constant $\mathrm{T}$ (i.e. Ii, $\mathrm{j}<\mathrm{T}$ ), each pixel on an image is substituted with a black one, and when Ii, $\mathrm{j}$ becomes greater than the constant $\mathrm{T}$ (i.e. $\mathrm{Ii}, \mathrm{j}<\mathrm{T}$ ), every pixel of the image is substituted by a pixel of a white color. This $\mathrm{T}$ is nothing but a user-specified threshold according to the object visualization requirement. The clear result of the thresholding technique is presented in fig 3.a, and the image on the left represents the original CT image as the input image.

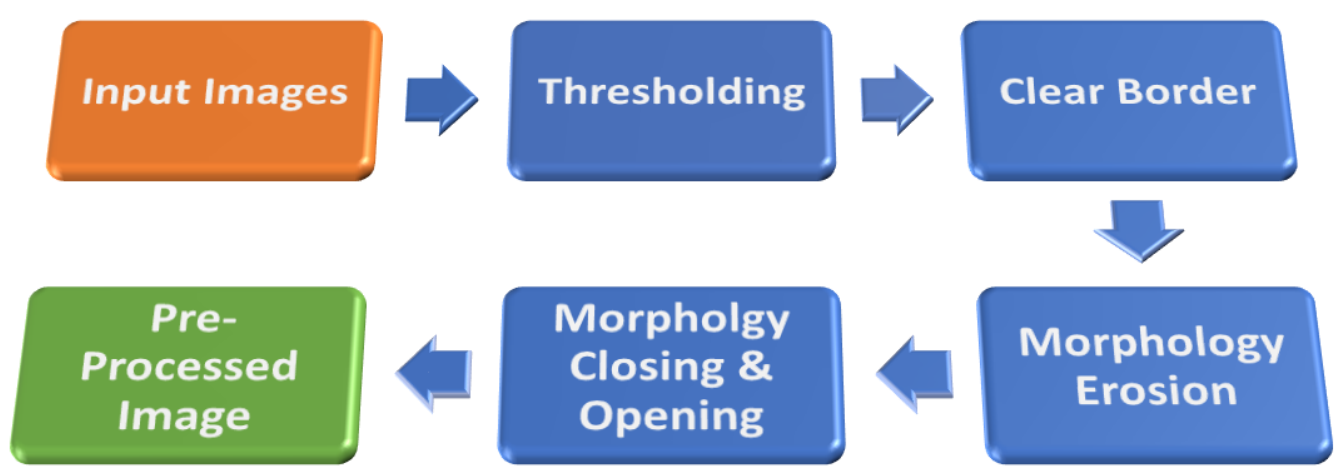

Fig 2: Pre-Processing Techniques for Lung Nodule Detection

\subsubsection{Thresholding}

Thresholding is the image processing technique used for transforming gray images into binary images. Pixel Ii, j tested for intensity with the simplest threshold methods. When $\mathrm{Ii}, \mathrm{j}$ is lesser than several fixed constant T (i.e. Ii, $\mathrm{j}<\mathrm{T}$ ), each pixel on an image is substituted with a black one, and when Ii, $\mathrm{j}$ becomes greater than the constant $\mathrm{T}$ (i.e. Ii, $\mathrm{j}<\mathrm{T}$ ), every pixel of the image is substituted by a pixel of a white color. This $\mathrm{T}$ is nothing but a user-specified threshold according to the object visualization requirement. The clear result of the thresholding technique is presented in fig 3.a, and the image on the left represents the original CT image as the input image.

\subsubsection{Clear Border}

The objects that are related to the image boundary must be removed after thresholding because they are image non-ROI objects. For extracting these items, a clear border approach was used. It also suppresses lighter-than-circle systems. The result is shown in Fig 3.b after clearing the border.

\subsubsection{Morphology Erosion}

An innovative dualistic image $\mathrm{e}=\mathrm{f} \Theta \mathrm{s}$ function is produced with the erosion of a dualistic image $f$ by an arranging element s. (denoted as "f $\ominus s ")$. When putting s on $\mathrm{f}, \mathrm{e}(\mathrm{x}, \mathrm{y})=$ 1 or else, $e(x, y)=0$, repeating all coordinates of the pixel ( $x$, $\mathrm{y}$ ) when placing $\mathrm{s}$ on $\mathrm{f}$. Minor details are removed when holes and gap between various region become larger. Fig 3.c indicates the result of erosion. 


\subsubsection{Morphology Closing and Opening}

A dilation pursued through erosion is the closing of an image $\mathrm{f}$ by an arranging element $\mathrm{s}$ (denotes $\mathrm{f} \bullet \mathrm{s}$ ). An innovative dualistic image $\mathrm{e}=\mathrm{f} \ominus \mathrm{s}$ is produced by the dilation from the image $f$ by an arranging element $s$. If element structure is hit by $f, e(x, y)=1$ or $e(x, y)=0$, for each coordinate of pixel $(x$, $y)$. This summates a layer of pixels both to the interior and exterior borders of areas to the opposite effect of erosion. So, dilation is used to increase the object's size. The holes formed by one region and the differences among the various areas get narrower after closing operation, and minor intrusions into the borders of a region are covered. Fig 3.d shows the result after closing. The morphology opening image is opened by a structuring element, accompanied by erosion and dilation. Also known as a filling method, it may open a gap among objects which are linked by a thin pixel bridge. Any region affected by erosion shall be restored by the dilation operation to its original size. The outcome of the opening image is shown in Fig 3.e.

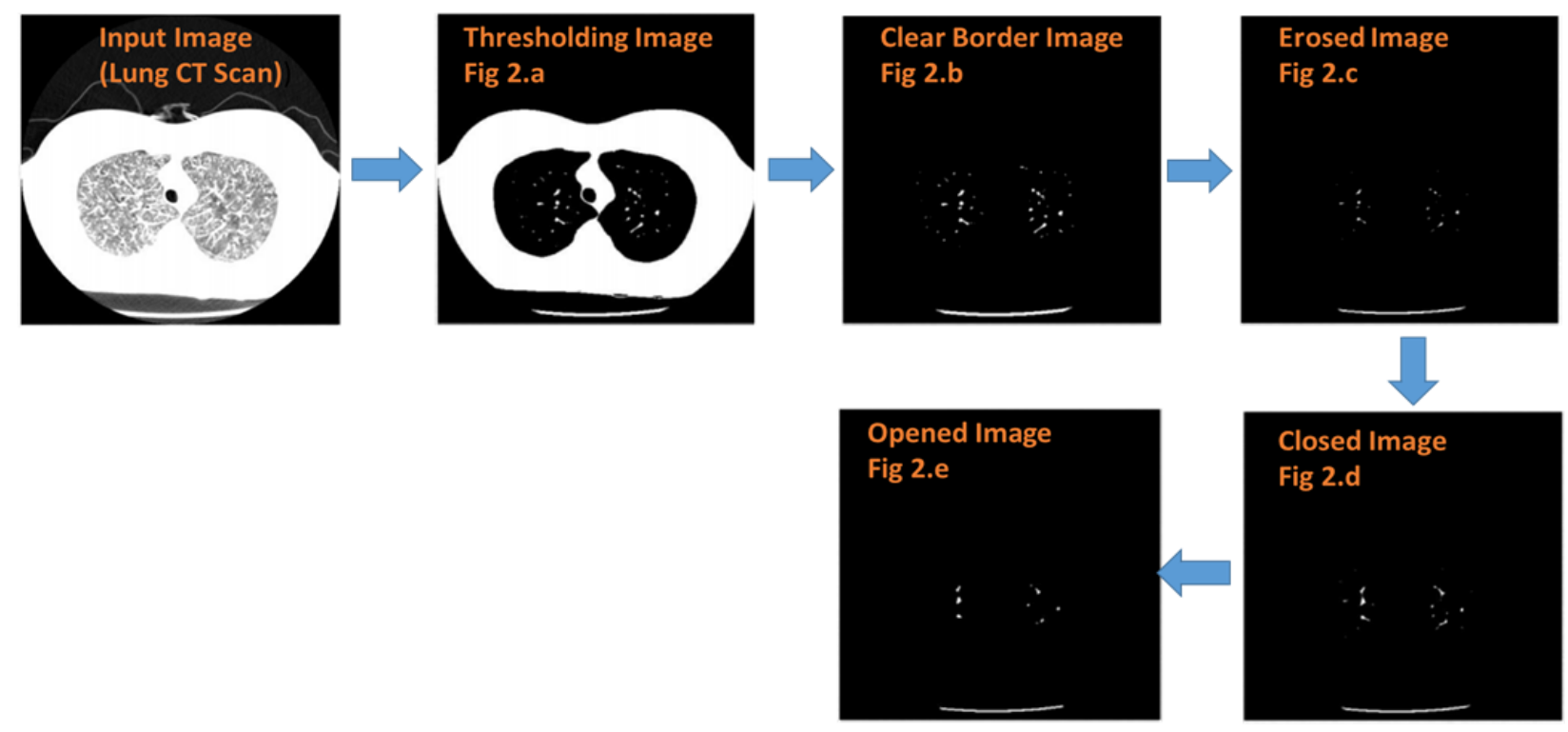

Fig 3: Results of Detection Algorithm from fig "a-e"

\subsection{Lung Nodule Classification}

In the proposed CMM, the classification part for CT scans using Multi-Output DenseNet for the lung nodules is illustrated. There are hundreds of $2 \mathrm{D}$-slices in a standard CT scan, e.g., a dimension of $512 \times 512$ gray images. Due to limited memory resources, direct handling of the entire 3D image scan is not efficient for a densenet. However, in the selected image data, most of the lung nodules contain less than ten slices, and some of the nodules are too scarce to discern between them when the whole 3D image is presented so, the MOD technique concentrates on the region where the nodule is present. Next, the architecture of MOD is expressed.

\subsubsection{Multi-Output DenseNet (MOD)}

Another way of shortening the range from input to output is DenseNet proposed in [1], which has two inputs and just one output. They can attain an improved result, due to the vanishing gradient is enhanced through the layer-closer links towards the output. Thus, the obtain technique from DenseNet is modified and advances 2D neural standard network by adding dense blocks that have direct connections amongst all block layers. Thus the 3D dual path is introduced with multiple output DenseNet. The architecture is composed of each path of five dense blocks. The first 3 dense components are composed of 4,10 , and 20 convolutional layers. The architecture of the MOD model is shown in figure 3. There are 12 feature maps in each convolution layer with 3D filters of dimension $3 \times 3 \times 3$. The last two dense blocks are composed of 20 convolutionary layers with 24 \& 48 feature maps that also create $3 \mathrm{D}$ convolutional filters about the size of
$3 \times 3 \times 3$, respectively. In order to achieve the best possible result, we present initial outputs that provide instant feedback from initial error assessment.

After every pooling layer that pursues the dense blocks in both pathways, the initial outputs were prepared in the designed model. The feature maps of both pathways from the last convolutionary layers are merged before every intermediate output and then referred to the classifier for the final output.

\section{EXPERIMENTS}

\subsection{Dataset}

The CMM was evaluated on the LIDC-IDRI public dataset, which contains $1110 \mathrm{CT}$ scans with radiologist annotations. One or more lung nodules are included in each image. The list of nodules mentioned in [3] is used to attain the $x, y$, and slice amount of the place of a nodule utilized to construct 3D image patches by way of the network input. Aimed at every nodule, we set a grade between 1-5. A zero grade demonstrates a diagnosis that is unavailable and unnoticed. Median grade values intended for a nodule are measured, then a median rating of less than 3 is regarded as benign besides 3 is used as malignant. Those nodules of an average value of 3 were removed. As a consequence, 808 lung nodules were obtained with special observations. In contrast, due to misplaced slices or non-uniform slice size, certain lung nodules are discarded. Ultimately, for testing, 790 lung nodules have been taken in which there is $44 \%$ benign and $57 \%$ malignant) 


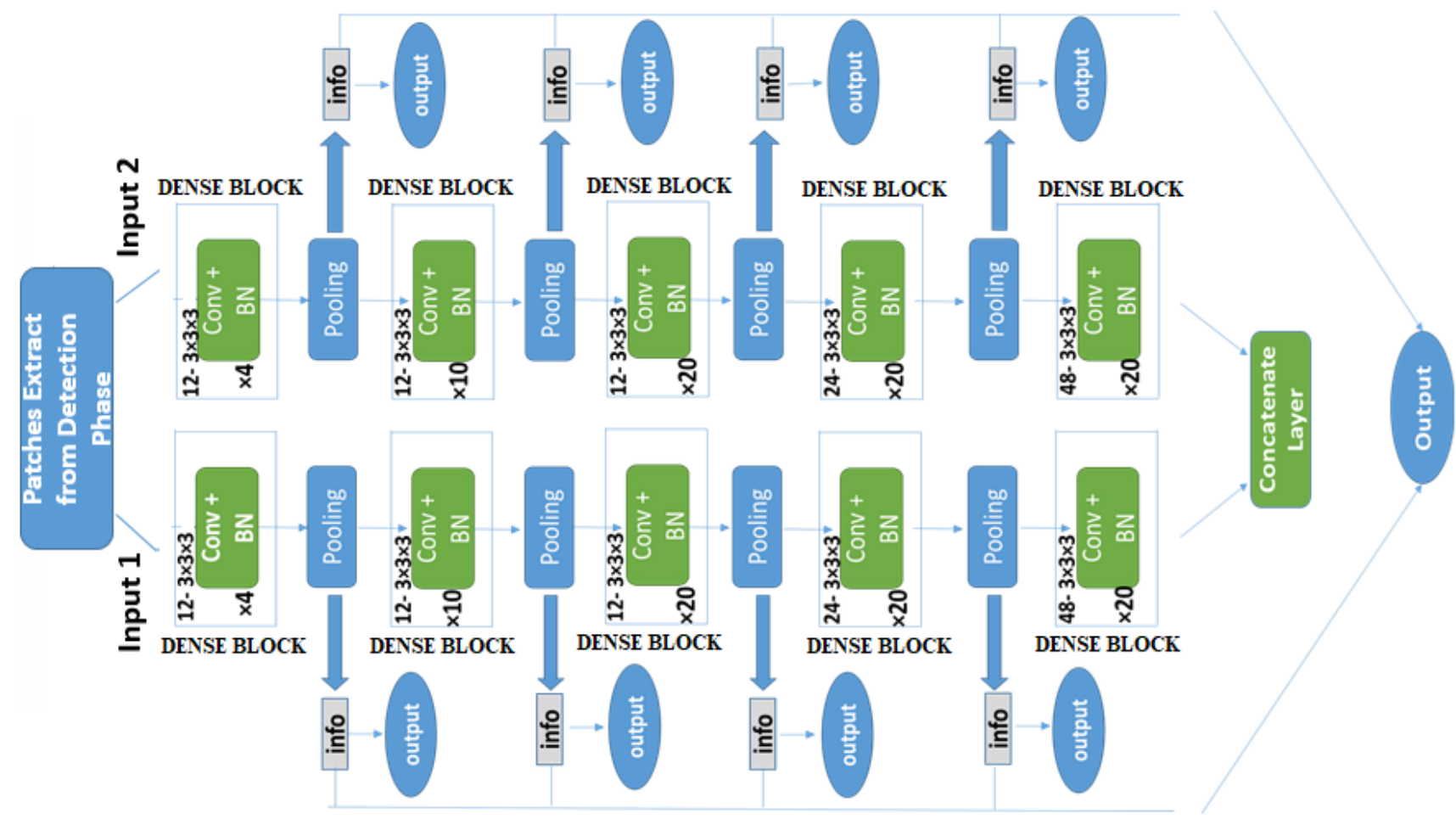

Fig 3: The architecture of the MOD model

\subsection{Results and Analysis}

With the same experimental conditions, the proposed Consignable Multi-Model (CMM) was tested against the Multi-Output DenseNet (MOD), which includes the same training set, initial parameters including learning rate scheme. The results of the CMM model on the LIDC-IDRI dataset are shown in Table 1 and its ROC curves in Fig 4. The CMM achieve the best accuracy of $92.10 \%$ and the AUC of 0.9680 , while MOD obtain an accuracy of $90.04 \%$ and AUC of 0.9544 . With early outputs link between convolutional layers, the method of shortening the input and output distance supports by the optimization and leads to improved optimal outcomes. On all major categories and the minor categories, the CMM performs a relatively accurate classification. To illustrate the classification of proposed CMM, it is also compared with some other popular methods that have a recent good performance which is presented in table 2. In [20], Wozniak et al. used a method that starts with the localization and extracting the lung nodules by measuring the local variance with the same size as the original pixel for each image. Shen et al. [19] have suggested a McCNN (Multi-crop CNN) network, a multi-scale input network for the classification of a lung nodule. A ML-CNN model is used by Lyu et al. [21] to classify between benign and malignant. In the pulmonary nodule CT images, ML-CNN consists of 3 CNNs to extract multi-scale capabilities. They also flatten the last pooling layer output into a single vector for each level and then tie it up. Yang et al. [22] used Unet, a complete machine system, to extract features rather than the traditional convolutionary neural network. Resnet was used to construct a hierarchy of extraction, and Adaboost was used to achieve the highest accuracy in unified reading, which was reached at $72.6 \%$. Convolutional neural network in [23] has used in which the functions are extracted hierarchically from the input image and ultimately incorporated into a fully connected layer with a softmax activation feature to identify the Nodules by four classes of convolution and max pooling layers. In the classification of nodules [24] (Nibali et al. 2017), the ResNet18 approach was used to show its usefulness, which has achieved great popularity in the area of computer vision. In [25] Multi-scaling features are derived via a multiconvolution system and the residual block structure allows the network to capture more high-level and semantine data. In addition, a technique is introduced to combine the last avgpooling surface characteristics and the last residual frame characteristics to further boost design quality.

Table 1. Results of CMM and MOD on LIDC-IDRI dataset. TPR stands for True positive rate or sensitivity, TNR represent true negative rate or specificity, PPV indicate positive predictive value or precision, AUC mean area under the ROC curve and ACC symbolize the accuracy, the percentage of correct predictions

\begin{tabular}{|c|c|c|c|c|c|}
\hline Network & $\begin{array}{c}\text { TPR } \\
\mathbf{\%}\end{array}$ & $\begin{array}{c}\text { TNR } \\
\mathbf{\%}\end{array}$ & $\begin{array}{c}\text { PPV } \\
\mathbf{\%}\end{array}$ & AUC & $\begin{array}{c}\text { ACC } \\
\mathbf{\%}\end{array}$ \\
\hline $\begin{array}{c}\text { Multi-Output } \\
\text { DenseNet } \\
\text { (MOD) }\end{array}$ & 90.36 & 90.24 & 90.44 & 0.9544 & 90.04 \\
\hline $\begin{array}{c}\text { Consignable } \\
\text { Multi-Model } \\
\text { (CMM) }\end{array}$ & 92.55 & 92.44 & 92.68 & 0.9680 & 92.10 \\
\hline
\end{tabular}




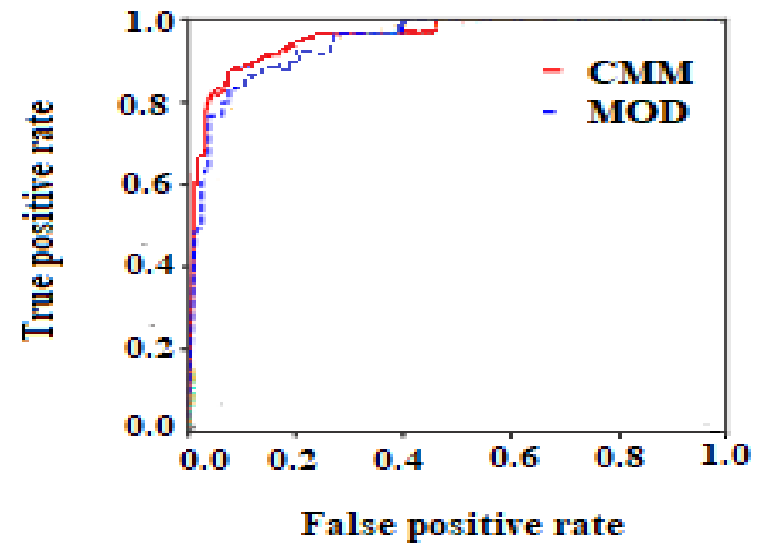

Fig 4: ROC curve of CMM technique

Table 2. Comparison of proposed CMM with other stateof-art methods

\begin{tabular}{|c|c|}
\hline Methods & $\begin{array}{c}\text { Classification } \\
\text { Accuracy }\end{array}$ \\
\hline CMM & $\mathbf{9 2 . 1 0}$ \\
\hline Wozniak et al. & 92.0 \\
\hline Shen et al. & 87.14 \\
\hline Guokai et al. & 87.5 \\
\hline Lyu et al. & 84.81 \\
\hline Yanf et al. & 72.6 \\
\hline
\end{tabular}

\section{CONCLUSION}

In this paper, the knowledge and preferences of doctors are used to strengthen the Consignable Multi-Model (CMM) algorithm, focusing on the limitations of existing diagnostics approaches for benign and malignant pulmonary nodules suggested by researchers and radiologists. So, we proposed the CMM algorithm that contain 2 components, i-e. Detection and Classification part. Moreover, lung nodule is first to identify in detection phase with different machine learning techniques and then Multi-Output DenseNet (MOD) accept the patches from the previous phase with its two input system and then classify the lung nodules using initial outputs. The CMM algorithm was evaluated on the LIDC-IDRI dataset; comparative to the architectures of other researchers, the presented algorithm is believed to have better performance having accuracy $92.10 \%$. Experimental results indicate that the CMM achieves the state-of-art in the diagnosis and classification of lung nodule issues. Future work may entail automated lung nodule detection, which relaxes manual annotations criteria for positions in the nodule.

\section{REFERENCES}

[1] Huang, Gao, et al. "Densely connected convolutional networks." Proceedings of the IEEE conference on computer vision and pattern recognition. 2017.

[2] Bengio, Yoshua. "Deep learning of representations for unsupervised and transfer learning." Proceedings of ICML workshop on unsupervised and transfer learning. 2012.
[3] Reeves, Anthony P., and Alberto M. Biancardi. "The lung image database consortium (lidc) nodule size report." (2011): 10-27.

[4] Siegel, Rebecca L., Kimberly D. Miller, and Ahmedin Jemal. "Cancer statistics, 2015." CA: a cancer journal for clinicians65.1 (2015): 5-29.

[5] Bach, Peter B., et al. "Benefits and harms of CT screening for lung cancer: a systematic review." Jama 307.22 (2012): 2418-2429.

[6] National Lung Screening Trial Research Team. "Reduced lung-cancer mortality with low-dose computed tomographic screening." New England Journal of Medicine 365.5 (2011): 395-409.

[7] Armato III, Samuel G., et al. "Data from lidc-idri. The cancer imaging archive." DOI http://doi. org/10.7937 K9 (2015): 7

[8] Javaid, Muzzamil, et al. "A novel approach to CAD system for the detection of lung nodules in CT images." Computer methods and programs in biomedicine 135 (2016): 125-139.

[9] Valente, Igor Rafael S., et al. "Automatic 3D pulmonary nodule detection in CT images: a survey." Computer methods and programs in biomedicine 124 (2016): 91107.

[10] Krishnamurthy, Senthilkumar, Ganesh Narasimhan, and Umamaheswari Rengasamy. "Three-dimensional lung nodule segmentation and shape variance analysis to detect lung cancer with reduced false positives." Proceedings of the Institution of Mechanical Engineers, Part $\mathrm{H}$ : Journal of Engineering in Medicine 230.1 (2016): 58-70.

[11] Liu, Ying, et al. "Radiological image traits predictive of cancer status in pulmonary nodules." Clinical Cancer Research 23.6 (2017): 1442-1449.

[12] Shewaye, Tizita Nesibu, and Alhayat Ali Mekonnen. "Benign-malignant lung nodule classification with geometric and appearance histogram features." arXiv preprint arXiv: 1605.08350 (2016).

[13] Shen, Wei, et al. "Multi-scale convolutional neural networks for lung nodule classification." International Conference on Information Processing in Medical Imaging. Springer, Cham, 2015

[14] Nibali, Aiden, Zhen He, and Dennis Wollersheim. "Pulmonary nodule classification with deep residual networks." International journal of computer assisted radiology and surgery 12.10 (2017): 1799-1808.

[15] Liu, Kui, and Guixia Kang. "Multiview convolutional neural networks for lung nodule classification." International Journal of Imaging Systems and Technology 27.1 (2017): 12-22.

[16] $\mathrm{Wu}$, Yifan, et al. "Automatic Mass Detection from Mammograms with Region-Based Convolutional Neural Network." Chinese Conference on Image and Graphics Technologies. Springer, Singapore, 2018.

[17] Dey, Raunak, Zhongjie Lu, and Yi Hong. "Diagnostic classification of lung nodules using 3D neura networks." 2018 IEEE 15th International Symposium on Biomedical Imaging (ISBI 2018). IEEE, 2018. 
[18] Huang, Xiaojie, Junjie Shan, and Vivek Vaidya. "Lung nodule detection in CT using 3D convolutional neural networks." 2017 IEEE 14th International Symposium on Biomedical Imaging (ISBI 2017). IEEE, 2017.

[19] Shen, Wei, et al. "Multi-crop convolutional neural networks for lung nodule malignancy suspiciousness classification." Pattern Recognition 61 (2017): 663-673.

[20] Woźniak, Marcin, et al. "Small lung nodules detection based on local variance analysis and probabilistic neural network." Computer methods and programs in biomedicine 161 (2018): 173-180..

[21] Lyu, Juan, and Sai Ho Ling. "Using multi-level convolutional neural network for classification of lung nodules on CT images." 2018 40th Annual International Conference of the IEEE Engineering in Medicine and Biology Society (EMBC). IEEE, 2018.
[22] Yang, et al. "Multi-classifier detection of lung nodules based on convolutional neural network." 2018 International Conference on Image and Video Processing, and Artificial Intelligence. Vol. 10836. International Society for Optics and Photonics, 2018.

[23] Yang, He, Hengyong Yu, and Ge Wang. "Deep learning for the classification of lung nodules." arXiv preprint arXiv:1611.06651(2016).

[24] Nibali, Aiden, Zhen He, and Dennis Wollersheim. "Pulmonary nodule classification with deep residual networks." International journal of computer assisted radiology and surgery 12.10 (2017): 1799-1808.

[25] Zhang, Guokai, et al. "Multi-scale pulmonary nodule classification with deep feature fusion via residual network." Journal of Ambient Intelligence and Humanized Computing (2018): 1-12. 